H. Fujimoto

Nagoya Math. J.

Vol. 124 (1991), 13-40

\title{
MODIFIED DEFECT RELATIONS FOR THE GAUSS MAP OF MINIMAL SURFACES, III
}

\author{
HIROTAKA FUJIMOTO
}

Dedicated to Professor N. Tanaka for his 60 th birthday

\section{$\S 1$. Introduction}

In [5], the author proved that the Gauss map of a nonflat complete minimal surface immersed in $\mathbf{R}^{3}$ can omit at most four points of the sphere, and in [7] he revealed some relations between this result and the defect relation in Nevanlinna theory on value distribution of meromorphic functions. Afterwards, Mo and Osserman obtained an improvement of these results in their paper [11], which asserts that if the Gauss map of a nonflat complete minimal surface $M$ immersed in $\mathbf{R}^{3}$ takes on five distinct values only a finite number of times, then $M$ has finite total curvature. The author also gave modified defect relations for holomorphic maps of a Riemann surface with a complete conformal metric into the $n$-dimensional complex projective space $\mathbf{P}^{n}(\mathbf{C})$ and, as its application, he showed that, if the (generalized) Gauss map $G$ of a complete minimal surface $M$ immersed in $\mathbf{R}^{m}$ is nondegenerate, namely, the image $G(M)$ is not contained in any hyperplane in $\mathrm{P}^{m-1}(C)$, then it can omit at most $m(m+1) / 2$ hyperplanes in general position ([8]). Here, the number $m(m+1) / 2$ is best-possible for arbitrary odd numbers and some small even numbers $m$ (see [6]). Recently, $\mathrm{Ru}$ showed that the "nondegenerate" assumption of the above result can be dropped ([13]). In this paper, we shall introduce a new definition of modified defect and prove a refined Modified defect relation. As its application, we shall give some improvements of the above-mentioned results in [5], [7], [8], [11] and [13].

We roughly explain here the modified defect relations given in this paper. More precise statements are given in $\S 5$. Let $M$ be an open Riemann surface with a conformal metric $d s^{2}$ and consider a nondegenerate

Received June 30, 1990. 
holomorphic map $f$ of $M$ into $\mathbf{P}^{n}(\mathbf{C})$. We denote by $\Omega_{f}$ the pull-back of Fubini-Study metric on $\mathbf{P}^{n}(\mathbf{C})$ through $f$, and by $f^{*}(H)^{[n]}$ the $n$-truncated pull-back of a hyperplane $H$ in $\mathbf{P}^{n}(\mathbf{C})$. In some cases, we regard them as $(1,1)$-currents on $M$.

We define the modified $\mathrm{H}$-defect of $H$ for $f$ by

$$
\begin{aligned}
D_{f}(H):=1-\inf \left\{\eta>0 ; f^{*}(H)^{[n]}\right. & \prec \eta \Omega_{f} \text { on } M-K \\
& \text { for some compact set } K\} .
\end{aligned}
$$

Here, by $\Omega_{1} \prec \Omega_{2}$ we mean that there are a divisor $\nu$ and a bounded real-valued function $k$ with mild singularities such that $\nu \geq c$ on the support $|\nu|$ of $\nu$ for a positive constant $c$ and

$$
\Omega_{1}+[\nu]=\Omega_{2}+d d^{c} \log k^{2}
$$

holds as currents, where [ $\nu$ ] denotes the current corresponding to $\nu$.

We also define the H-order of $f$ by

$$
\rho_{f}:=\inf \left\{\rho>0 ;-\operatorname{Ric}_{d s^{2}} \prec \rho \Omega_{f} \text { on } M-K \text { for some compact set } K\right\} \text {. }
$$

After Chen [2] we say that hyperplanes $H_{j}(1 \leq j \leq q)$ are located in $N$-subgeneral position if $H_{j_{0}} \cap \cdots \cap H_{j_{N}}=\varnothing$ for all $1 \leq j_{0}<\cdots<j_{N} \leq q$, where $q>N \geq n$. Particularly, we say that $H_{j}(1 \leq j \leq q)$ are in general position if they are in $n$-subgeneral position.

The modified defect relation proved in this paper is stated as follows:

TheOREM 1.1. Let $M$ be an open Riemann surface with a complete conformal metric $d s^{2}$, and let $f$ be a nondegenerate holomorphic map of $M$ into $\mathbf{P}^{n}(\mathbf{C})$ For the particular case where $M$ is biholomorphic with a compact Riemann surface $\bar{M}$ with finitely many points removed, we assume that $f$ cannot be extended to a holomorphic map of $\bar{M}$ into $\mathbf{P}^{n}(\mathbf{C})$. Then, for arbitrary hyperplanes $H_{1}, \cdots, H_{q}$ in $\mathbf{P}^{n}(\mathbf{C})$ located in $N$-subgeneral position,

$$
\sum_{j=1}^{q} D_{f}\left(H_{j}\right) \leq(2 N-n+1)\left(1+\frac{\rho_{f} n}{2}\right)
$$

Let $M$ be a (connected, oriented) minimal surface immersed in $\mathbf{R}^{m}$. We can regard $M$ as an open Riemann surface with a conformal metric. The set of all oriented 2-planes in $\mathbf{R}^{m}$ can be identified with the quadric

$$
Q_{m-2}(\mathbf{C})=\left\{\left(w_{1}: \cdots: w_{m}\right) ; w_{1}^{2}+\cdots+w_{m}^{2}=0\right\}\left(\subseteq \mathbf{P}^{m-1}(\mathbf{C})\right) .
$$


By definition, the Gauss map of $M$ is the map $G: M \rightarrow Q_{m-2}(\mathbf{C})\left(\subset \mathbf{P}^{m-1}(\mathbf{C})\right)$ which maps each $p \in M$ to the point in $Q_{m-2}(\mathbf{C})$ corresponding to the oriented tangent plane of $M$ at $p$. By the assumption of $M, G$ is a holomorphic map of $M$ into $\mathbf{P}^{m-1}(\mathbf{C})$. We can conclude from Theorem 1.1 the following:

THEOREM 1.3. Let $M$ be a nonflat complete minimal surface immersed in $\mathbf{R}^{m}$ with infinite total curvature, and let $G$ be the Gauss map of $M$. Then, for arbitrary hyperplanes $H_{1}, \cdots, H_{q}$ in $\mathbf{P}^{m-1}(\mathbf{C})$ located in general position,

$$
\sum_{j=1}^{q} G_{G}\left(H_{j}\right) \leq \frac{m(m+1)}{2} .
$$

For a holomorphic map $f$ of an open Riemann surface $M$ into $\mathbf{P}^{n}(\mathbf{C})$ and a hyperplane $H$ in $\mathbf{P}^{n}(\mathbf{C})$ we can show that $D_{f}(H)=1$ if $f^{-1}(H)$ is finite. This yields the following improvement of a result of $\mathrm{Ru}$ ([13]):

CoRollary 1.4. Let $M$ be a nonflat complete minimal surface immersed in $\mathbf{R}^{m}$ with infinite total curvature, and let $G$ be the Gauss map of $M$. If $G^{-1}\left(H_{j}\right)$ are finite for $q$ hyperplanes $H_{1}, \cdots, H_{q}$ in $\mathbf{P}^{m-1}(\mathbf{C})$ located in general position, then $q \leq m(m+1) / 2$.

In the case $m=3, Q_{1}(\mathbf{C})$ can be identified with $\mathbf{P}^{1}(\mathbf{C})$. The Gauss map $G$ of $M$ is considered as a holomorphic map of $M$ into $\mathbf{P}^{1}(\mathbf{C})$ with $\rho_{G} \leq 2$. Theorem 1.1 implies the above-mentioned result given by Mo and Osserman.

In this paper, we also consider minimal surface with branch points. We introduce the new notion of branching H-order for minimal surfaces and give modified defect relations for the Gauss map which relate to this notion.

The author wishes to thank Professor I. Wakabayashi for his useful critical comments.

\section{§ 2. Nochka weights for hyperplanes in subgeneral position}

For later use, we recall an algebraic theorem given by Nochka in [12] (cf. [2]).

We consider $q$ hyperplanes $H_{j}(1 \leq j \leq q)$ in $\mathbf{P}^{n}(\mathbf{C})$ which are given by

$$
H_{j}:\left(A_{j}, W\right)=0 \quad(1 \leq j \leq q)
$$


for $A_{j} \in \mathbf{C}^{n+1}-\{0\}$, where $q>N \geq n$ and $(A, W)$ means $a_{0} w_{0}+\cdots+a_{n} w_{n}$ for a vector $A=\left(a_{0}, \cdots, a_{n}\right)$ and homogeneous coordinates $W=\left(w_{0}: \cdots: w_{n}\right)$. After Chen ([2]), we give the following definition.

Definition 2.1. We say that hyperplanes $H_{1}, \cdots, H_{q}$ (or vectors $A_{1}$, $\left.\cdots, A_{q}\right)$ are in $N$-subgeneral position if, for every $1 \leq j_{0}<\cdots<j_{N} \leq q$, $A_{j_{0}}, A_{j_{1}}, \cdots, A_{j_{N}}$ generate $\mathbf{C}^{n+1}$, or equivalently,

$$
H_{j_{0}} \cap \cdots \cap H_{j_{N}}=\varnothing \text {. }
$$

We assume that $H_{1}, \cdots, H_{q}$ are in $N$-subgeneral position in the following.

Set $Q:=\{1,2, \cdots, q\}$. For each $R \subseteq Q$ we denote by $\sharp R$ the number of elements of $R$ and by $d(R)$ the dimension of the vector subspace of $\mathbf{C}^{n+1}$ generated by $\left\{A_{j} ; j \in R\right\}$. For convenience sake, we set $d(\varnothing)=0$.

In [12], Nochka has given the following theorem to prove Cartan's conjecture.

Theorem 2.2. Let $H_{1}, H_{2}, \cdots, H_{q}$ be hyperplanes in $\mathbf{P}^{n}(\mathbf{C})$ located in $N$-subgeneral position, where $q>2 N-n+1$. Then there are some constants $\omega(1), \cdots, \omega(q)$ and $\theta$ satisfying the following conditions;

(i) $0<\omega(j) \leq \theta \leq 1 \quad(1 \leq j \leq q)$,

(ii) $\sum_{j=1}^{q} \omega(j)=n+1+\theta(q-2 N+n-1)$,

(iii) $\frac{n+1}{2 N-n+1} \leq \theta \leq \frac{n+1}{N+1}$,

(iv) if $R \subset Q$ and $0<\sharp R \leq N+1$, then $\sum_{j \in R} \omega(j) \leq d(R)$.

For the proof, see [12] or ]2].

Definition 2.3. We call constants $\omega(j)(1 \leq j \leq q)$ and $\theta$ with the properties (i) (iv) Nochka weights and a Nochka constant for $H_{1}, \cdots, H_{q}$ respectively.

By definition, $H_{j}(1 \leq j \leq q)$ are in general position if and only if they are in $n$-subgeneral position. If $H_{1}, \cdots, H_{q}$ are in general position, then we have necessarily $\omega(1)=\cdots=\omega(q)=\theta=1$.

We give here a property of Wronskians relating to Nochka weights. Let $f_{0}, \cdots, f_{n}$ be holomorphic functions without common zeros on a domain $D$ in $\mathbf{C}$. We denote the Wronskian of $f_{0}, \cdots, f_{n}$ by

$$
W\left(f_{0}, \cdots, f_{n}\right)=\operatorname{det}\left(f_{m}^{(l)} ; 0 \leq l, m \leq n\right) .
$$


Assume that $f_{0}, \cdots, f_{n}$ are linearly independent over $\mathbf{C}$, or $W\left(f_{0}, \cdots, f_{n}\right)$ $\not \equiv 0$. Consider $q$ vectors

$$
A_{j}=\left(a_{j 0}, a_{j 1}, \cdots, a_{j n}\right) \in \mathbf{C}^{n+1} \quad(1 \leq j \leq q)
$$

located in $N$-subgeneral position and take Nochka weights $\omega(j)(1 \leq j \leq q)$, where $q>2 N-n+1$. Set

$$
F_{j}=a_{j 0} f_{0}+\cdots+a_{j n} f_{n} \quad(1 \leq j \leq q) .
$$

For a nonzero meromorphic function $\psi$ on $D$ we define the map $\nu_{\psi}: D \rightarrow \mathbf{Z}$ by

$$
\nu_{\psi}(\zeta):=\text { the order of the meromorphic function } \psi \text { at } \zeta .
$$

Consider a function

$$
\varphi=\frac{\left|W\left(f_{0}, \cdots, f_{n}\right)\right|}{\left|F_{1}\right|^{\omega(1)} \cdots\left|F_{q}\right|^{\omega(q)}}
$$

and set

$$
\nu_{\varphi}=\nu_{W}-\sum_{j=0}^{q} \omega(j) \nu_{F_{j}}
$$

where $W=W\left(f_{0}, \cdots, f_{n}\right)$.

LEMMA 2.4. In the above situation, we have

$$
\nu_{\varphi}+\sum_{j=1}^{4} \omega(j) \min \left(\nu_{F_{j}}, n\right) \geq 0 .
$$

Proof. It suffices to show that

$$
\nu_{W} \geq \sum_{j=1}^{q} \omega(j)\left(\nu_{F_{j}}-n\right)^{+},
$$

where $x^{+}$means $\max (x, 0)$ for a real number $x$. In fact, since

$$
\min \left(\nu_{F_{j}}, n\right)+\left(\nu_{F_{j}}-n\right)^{+}=\nu_{F_{j}},
$$

we can conclude from (2.5)

$$
\begin{gathered}
\nu_{\varphi}+\sum_{j=0}^{q} \omega(j) \min \left(\nu_{F_{j}}, n\right)=\nu_{W}-\sum_{j=1}^{q} \omega(j) \nu_{F_{j}}+\sum_{j=1}^{q} \omega(j) \min \left(\nu_{F_{j}}, n\right) \\
\geq \sum_{j=1}^{q} \omega(j)\left(\left(\nu_{F_{j}}-n\right)^{+}+\min \left(\nu_{F_{j}}, n\right)-\nu_{F_{j}}\right)=0 .
\end{gathered}
$$

To prove (2.5), take a point $\zeta \in D$, and set $Q:=\{1,2, \cdots, q\}$ and 


$$
S:=\left\{j \in Q ; \nu_{F_{j}}(\zeta) \geq n+1\right\} .
$$

We may assume that $S \neq \varnothing$. Then, $\sharp S \leq N$. For, otherwise, by $N$-subgenerality of $A_{j}$ 's there are $n+1$ distinct $j_{0}, \cdots, j_{n} \in S$ such that $A_{j_{0}}, \cdots$, $A_{j_{n}}$ are linearly independent. So, $f_{0}, \cdots, f_{n}$ are represented as linear combinations of $F\left(H_{j_{0}}\right), \cdots, F\left(H_{j_{n}}\right)$, whence $f_{0}, \cdots, f_{n}$ have a common zero at $\zeta$. We now consider the sets $S_{\tau}(0 \leq \tau \leq t)$ such that

$$
S_{0}:=\varnothing \neq S_{1} \subset S_{2} \subset \cdots \subset S_{t}=S
$$

and for each $j \in S_{\tau}-S_{\tau-1} \nu_{F_{j}}(\zeta)$ equals some constant $m_{\tau}$, where $m_{1}>m_{2}>$ $\cdots>m_{t} \geq n+1$. Let $V(R)$ be the vector space generated by $\left\{A_{j} ; j \in R\right\}$ for each $R \subseteq Q$. Then,

$$
V\left(S_{1}\right) \subseteq V\left(S_{2}\right) \subseteq \cdots \subseteq V\left(S_{t}\right) .
$$

For each $\tau$ take a subset $T_{\tau}$ of $S_{\imath}$ such that $T_{\tau-1} \subset T_{\tau}$ and $\left\{A_{j} ; j \in T_{\tau}\right\}$ gives a basis of $V\left(S_{\tau}\right)$. We then have $\sharp\left(T_{\tau}-T_{\imath-1}\right)=d\left(S_{\tau}\right)-d\left(S_{\imath-1}\right)$. For brevity, we set $m_{\tau}^{*}:=m_{t}-n$. By the use of Theorem 2.2, (iv) we obtain

$$
\begin{aligned}
& \sum_{j=1}^{q} \omega(j)\left(\nu_{F_{j}}-n\right)^{+}=\sum_{j \in S} \omega(j)\left(\nu_{F_{j}}-n\right)^{+}=\sum_{\tau=1}^{t} \sum_{j \in S_{\tau}-S_{\tau}-1} \omega(j) m_{\tau}^{*} \\
& \quad=\sum_{\tau=1}^{t} \sum_{j \in S_{\tau-S_{\tau}-1}} \omega(j)\left(\left(\sum_{\sigma=\tau}^{t-1} m_{\sigma}^{*}-m_{\sigma+1}^{*}\right)+m_{t}^{*}\right) \\
& \quad=\left(m_{1}^{*}-m_{2}^{*}\right) \sum_{j \in S_{1}} \omega(j)+\left(m_{2}^{*}-m_{3}^{*}\right) \sum_{j \in S_{2}} \omega(j)+\cdots+m_{t}^{*} \sum_{j \in S_{t}} \omega(j) \\
& \quad \leq d\left(S_{1}\right)\left(m_{1}^{*}-m_{2}^{*}\right)+d\left(S_{2}\right)\left(m_{2}^{*}-m_{3}^{*}\right)+\cdots+d\left(S_{t}\right) m_{t}^{*} \\
& \quad=d\left(S_{1}\right) m_{1}^{*}+\left(d\left(S_{2}\right)-d\left(S_{1}\right)\right) m_{2}^{*}+\cdots+\left(d\left(S_{t}\right)-d\left(S_{t-1}\right)\right) m_{t}^{*} \\
& \quad=\# T_{1} m_{1}^{*}+\#\left(T_{2}-T_{1}\right) m_{2}^{*}+\cdots+\#\left(T_{t}-T_{t-1}\right) m_{t}^{*} .
\end{aligned}
$$

Set $T_{t}=\left\{j_{0}, \cdots, j_{k}\right\}$, where $k \leq n$. Since $A_{j_{0}}, \cdots, A_{j_{k}}$ are linearly independent, after a suitable nonsingular linear transformation of homogeneous coordinates, we may assume that $f_{0}=F_{j_{0}}, \cdots, f_{k}=F_{j_{k}}$. Then, by the Laplace' expansion theorem of determinants, $W$ is expanded as the sum of the products of some minors of degree $n-k$ and some minors of degree $k+1$ whose components consist of the $\leq n$-th derivatives of $F_{j_{0}}$, $\cdots, F_{j_{k}}$. This implies that

$$
\nu_{W}(\zeta) \geq \sum_{j \in T_{t}}\left(\nu_{F_{j}}(\zeta)-n\right)
$$

Since $\nu_{F_{j}}(\zeta)=m_{\imath}$ for every $j \in T_{\tau}-T_{\tau-1}$ this quantity coincides with the last term of the above inequalities. This completes the proof of Lemma 2.4 . 


\section{$\S 3$. Some properties of the contact functions for derived curves}

We recall some known results on the derived curves for a holomorphic map of an open Riemann surface $M$ into $\mathbf{P}^{n}(\mathbf{C})$.

Let $f: M \rightarrow \mathbf{P}^{n}(\mathbf{C})$ be a nondegenerate holomorphic map. Take a reduced representation $f=\left(f_{0}: \cdots: f_{n}\right)$ on $M$ with holomorphic functions $f_{i}$ on $M$ having no common zeros. Set $\|f\|=\left(\sum_{0 \leq j \leq n}\left|f_{i}\right|^{2}\right)^{1 / 2}$ and $F=$ $\left(f_{0}, \cdots, f_{n}\right)$. For each $p \in M$, taking a holomorphic local coordinate on some neighborhood $U$ of $p$, we set $F^{(l)}=\left(f_{0}^{(l)}, \cdots, f_{n}^{(l)}\right)$ for each $l=0,1$, $\cdots$, and define the map

$$
F_{k}:=F^{(0)} \wedge F^{(1)} \wedge \cdots \wedge F^{(k)}: U \longrightarrow \bigwedge^{k+1} \mathbf{C}^{n+1} \quad(0 \leq k \leq n-1),
$$

where $F_{0}=F^{(0)}:=F$. Let $\pi: \bigwedge^{k+1} \mathbf{C}^{n+1}-\{0\} \rightarrow \mathrm{P}^{N_{R}}(\mathbf{C})$ denote the canonical projection, where $N_{k}:=\left(\begin{array}{l}n+1 \\ k+1\end{array}\right)-1$. By definition, the $k$-th derived curve of $f$ is the map $f^{k}=\pi \cdot F_{k}$, which does not depend on the choice of a holomorphic local coordinate and so is well-defined on the totality of $M$. We locally define

$$
\left|F_{k}\right|=\left(\sum_{0 \leq j_{0}<\cdots<j_{k} \leq n}\left|W\left(f_{f_{0}}, \cdots, f_{j_{k}}\right)\right|^{2}\right)^{1 / 2} \quad(0 \leq k \leq n-1) .
$$

By definition, the pull-back $\Omega_{k}$ of the Fubini-Study metric on $\mathbf{P}^{N_{k}}(\mathbf{C})$ by $f^{k}$ is locally given by

$$
\Omega_{k}=d d^{c} \log \left|F_{k}\right|^{2},
$$

where $d^{c}=(\sqrt{-1} / 4 \pi)(\bar{\partial}-\partial)$. Take a hyperplane

$$
H:(A, W)=0,
$$

where $A=\left(a_{0}, \cdots, a_{n}\right)$ is a unit vector. Set

$$
\left|F_{k}(H)\right|^{2}=\left|F_{k} \vee A\right|^{2}:=\sum_{0 \leq i_{1}<\cdots<i_{k} \leq n}\left|\sum_{j \neq i_{1}, \cdots, i_{k}} a_{j} W\left(f_{j}, f_{i_{1}}, \cdots, f_{i_{k}}\right)\right|^{2},
$$

where $\vee$ denotes the interior product as in [14]. We define the contact functions

$$
\varphi_{k}(H)(z):=\frac{\left|F_{k}(H)(z)\right|^{2}}{\left|F_{k}(z)\right|^{2}},
$$

which are well-defined on the totality of $M$ and do not vanish identically. For $k=0$, we have $F_{0}(H)=F(H):=(A, F)$ and $\varphi_{0}(H)=|F(H)|^{2} /\|f\|^{2}$.

We now give the following so-called sums into products estimates. 
Theorem 3.1. Let $H_{1}, \cdots, H_{q}$ be hyperplanes in $\mathbf{P}^{n}(\mathbf{C})$ located in $N$ subgeneral position and $\omega(j)(1 \leq j \leq q)$ be Nochka weights for these hyperplanes. For an arbitrarily given $a>1$ and $0 \leq k \leq n-1$ set

$$
\Phi_{j k}:=\frac{\varphi_{k+1}\left(H_{j}\right)}{\varphi_{k}\left(H_{j}\right) \log ^{2}\left(a / \varphi_{k}\left(H_{j}\right)\right)} .
$$

Then, there exists a positive constant $C_{k}$ depending only on $k$ and $H_{j}$ such that

$$
\sum_{j=1}^{q} \omega(j) \Phi_{j k} \geq C_{k}\left(\prod_{j=1}^{q} \Phi_{j k}^{\omega(j)}\right)^{1 /(n-k)}
$$

on $M-\bigcup_{1 \leq j \leq q}\left\{z ; \varphi_{k}\left(H_{j}\right)(z)=0\right\}$.

For the case where $H_{j}$ are in general position, this is classical (cf., e.g., [4] or [14]). For the general case, the proof is given by similar arguments by the use of Theorem 2.2 (cf., [2]). Since the description of Chen is lengthy, we give here a shorter proof.

For the proof, we use the following elementary inequality.

$$
\begin{aligned}
& \text { For all positive number } x_{1}, \cdots, x_{n} \text { and } a_{1}, \cdots, a_{n} \text {, } \\
& \qquad a_{1} x_{1}+\cdots+a_{n} x_{n} \geq\left(a_{1}+\cdots+a_{n}\right)\left(x_{1}^{a_{1}} \cdots x_{n}^{a_{n}}\right)^{1 /\left(a_{1}+\cdots+a_{n}\right)} .
\end{aligned}
$$

Proof of Theorem 3.1. Let

$$
H_{j}:\left(W, A_{j}\right)=0 \quad(1 \leq j \leq q),
$$

where we choose $A_{j}$ with $\left|A_{j}\right|=1$ for each $j$.

We consider the set $\mathscr{R}_{k}$ of all subsets $R$ of $Q:=\{1,2, \cdots, q\}$ such that $\dot{d}(R) \leq n-k$. For each $P \in G(n, k)$ we take a nonzero decomposable $(k+1)$-vector $E$ such that $P=\left\{X \in \mathbf{C}^{n+1} ; E \wedge X=0\right\}$ and set

$$
\psi_{k}(P)=\max _{R \in x_{k}} \min \left\{\frac{\left|E \vee A_{j}\right|^{2}}{|E|^{2}} ; j \notin R\right\},
$$

which depends only on $P$. We regard $\psi_{k}$ as a function on the Grassmann manifold $G(n, k)$. For each nonzero vector $E=E_{0} \wedge \cdots \wedge E_{k}$ we set

$$
R:=\left\{j \in Q ; E \vee A_{j}=0\right\} .
$$

Since $E \vee A_{j}=0$ means that $A_{j}$ is contained in the orthogonal complement of the vector space generated by $E_{0}, \cdots, E_{k}$, we see $d(R) \leq n-k$, namely, $R \in \mathscr{R}_{k}$. This yields that $\psi_{k}$ is positive everywhere on $G(n, k)$. 
Since $\psi_{k}$ is continuous on the compact space $G(n, k)$, it has a positive lower bound $\delta$ on $G(n, k)$.

Take a point $z$ with $F_{k}(z) \neq 0$. Since $F_{k}(z)$ determines a point $P \in$ $G(n, k)$, there is a set $R$ in $\mathscr{R}_{k}$ such that $\varphi_{k}\left(H_{j}\right)=\left|E \vee A_{j}\right|^{2} /|E|^{2}=\psi_{k}(P)$ $\geq \delta$ for all $j \notin R$. Therefore, we can choose a finite positive constant $K$ depending only on $H_{j}$ 's such that $\Phi_{j k}(z) \leq K$ for all $j \notin R$. Set $S=$ $\left\{j ; \Phi_{j k}>K\right\}$. We may assume $S \neq \varnothing$. In fact, otherwise, we have

$$
\sum_{j=1}^{q} \omega(j) \Phi_{j k} \geq K l_{0}\left(\prod_{j=1}^{q}\left(\frac{\Phi_{j k}}{K}\right)^{\omega(j) / l_{0}}\right) \geq K l_{0}\left(\prod_{j=1}^{q}\left(\frac{\Phi_{j k}}{K}\right)^{\omega(j)}\right)^{1 /(n-k)}
$$

where $l_{0}:=\sum_{j=1}^{q} \omega(j)$. We see $S \subseteq R$ and so $d(S) \leq n-k$. Then, $l:=$ $\sum_{j \in S} \omega(j) \leq n-k$ by Theorem 2.2 , (iv) and $\sharp S \leq N$ by the assumption. By the help of (3.3) we obtain

$$
\begin{aligned}
\sum_{j=1}^{q} \omega(j) \Phi_{j k} & \geq \sum_{j \in S} \omega(j) \Phi_{j k} \geq K l \prod_{j \in S}\left(\frac{\Phi_{j k}}{K}\right)^{\omega(j) / l} \\
& \geq K l \prod_{j \in S}\left(\frac{\Phi_{j k}}{K}\right)^{\omega(j) /(n-k)} \geq C \prod_{j=1}^{q}\left(\frac{\Phi_{j k}}{K}\right)^{\omega(j) /(n-k)}
\end{aligned}
$$

for some constant $C$. Eventually, we can find a positive constant $C_{k}$ satisfying (3.2), which depends only on $k$ and $H_{1}, \cdots, H_{q}$. This gives Theorem 3.1.

Now, we give the following improvement of [8, Proposition 3.5].

Theorem 3.4. Let $H_{1}, \cdots, H_{q}$ be hyperplanes in $\mathbf{P}^{n}(\mathbf{C})$ located in $N$ subgeneral position and let $\omega(j)(1 \leq j \leq q)$ and $\theta$ be Nochka weights and a Nochka constant for these hyperplanes, where $q>2 N-n+1$. For every $\varepsilon>0$ there exist some positive numbers $a(>1)$ and $C$ depending only on $\varepsilon$ and $H_{j}(1 \leq j \leq q)$ such that

$$
\begin{aligned}
& d d^{c} \log \frac{\prod_{\left.\substack{n-1 \\
k=0} F_{k}\right|^{2 \varepsilon}}}{\prod_{1 \leq j \leq q, 0 \leq k \leq n-1} \log ^{2 \omega(j)}\left(a / \varphi_{k}\left(H_{j}\right)\right)} \\
& \quad \geq C\left(\frac{\left|F_{0}\right|^{2 \theta(q-2 N+n-1)}\left|F_{n}\right|^{2}}{\prod_{j=1}^{q}\left(\left|F\left(H_{j}\right)\right|^{2} \prod_{k=0}^{n-1} \log ^{2}\left(a / \varphi_{k}\left(H_{j}\right)\right)\right)^{\omega(j)}}\right)^{2 /(n(n+1))} \sqrt{-1} d z \wedge d \bar{z} .
\end{aligned}
$$

Proof. The proof is similar to that for the case where $H_{j}$ are in general position. We state here only its outline. As is proved in [4, p. 129] (or [14, p. 122]), for each positive $\varepsilon$ there exists a constant $a_{0}$ depending only on $\varepsilon$ such that for every hyperplane $H$ in $\mathbf{P}^{n}(\mathbf{C})$ and $a \geq a_{0}$

$$
d d^{c} \log \frac{1}{\log \left(a / \varphi_{k}(H)\right)} \geq \frac{\varphi_{k+1}(H)}{\varphi_{k}(H) \log ^{2}\left(a / \varphi_{k}(H)\right)} \Omega_{k}-\frac{\varepsilon}{l_{0}} \Omega_{k},
$$


where $l_{0}=\sum_{j=1}^{q} \omega(j)$ and $\Omega_{k}=d d^{c} \log \left|F_{k}\right|^{2}$. Choosing some $a \geq a_{0}$, we denote the left hand side of (3.5) by $A$. According to Theorem 3.1 and (3.3) we get

$$
\begin{aligned}
A & \geq \varepsilon \sum_{k=0}^{n-1} \Omega_{k}+\sum_{j=1}^{q} \omega(j) \sum_{k=0}^{n-1}\left(\frac{2 \varphi_{k+1}\left(H_{j}\right)}{\varphi_{k}\left(H_{j}\right) \log ^{2}\left(a / \varphi_{k}\left(H_{j}\right)\right)}-\frac{\varepsilon}{l_{0}}\right) \Omega_{k} \\
& =\sum_{k=0}^{n-1} 2\left(\sum_{j=1}^{q} \omega(j) \Phi_{j k}\right) \Omega_{k} \geq C_{1} \sum_{k=0}^{n-1}\left(\prod_{j=0}^{q} \Phi_{j k}^{\omega(j)}\right)^{1 /(n-k)} \Omega_{k} \\
& \geq C_{2} \prod_{k=0}^{n-1}\left(\prod_{j=1}^{q} \Phi_{j k}^{\omega(j)} \lambda_{k}^{2(n-k)}\right)^{2 /(n(n+1))} \sqrt{-1} d z \wedge d \bar{z}
\end{aligned}
$$

for some positive constants $C_{1}$ and $C_{2}$, where $\Omega_{k}=\lambda_{k}^{2} \sqrt{-1} d z \wedge d \bar{z}$. Observe

$$
\prod_{k=0}^{n-1} \Phi_{j k}=\frac{\left|F_{0}\right|^{2}}{\mid F\left(H_{j}\right)^{2}} \prod_{k=0}^{n-1} \frac{1}{\log ^{2}\left(a / \varphi_{k}\left(H_{j}\right)\right)}
$$

and

$$
\prod_{k=0}^{n-1} \lambda_{k}^{2(n-k)}=\frac{\left|F_{n}\right|^{2}}{\left|F_{0}\right|^{2(n+1)}}
$$

as in the previous paper $[8$, p. 373]. We obtain

$$
A \geq C\left(\frac{\left|F_{0}\right|^{2\left(l_{0}-n-1\right)}\left|F_{n}\right|^{2}}{\prod_{j=1}^{q}\left(\left|F\left(H_{j}\right)\right|^{2} \prod_{k=0}^{n-1} \log ^{2}\left(a / \varphi_{k}\left(H_{j}\right)\right)\right)^{\omega(j)}}\right)^{2 /(n(n+1))} \sqrt{-1} d z \wedge d \bar{z} .
$$

Since $l_{0}-n-1=\theta(q-2 N+n-1)$ by Theorem 2.2 , (ii), we can conclude Theorem 3.4.

\section{§4. Metrics with negative curvature}

Let $M$ be an open Riemann surface. We consider a function $u$ on $M$ possibly with singularities in a discrete subset of $M$.

Definition 4.1. We call $u$ a function with mild singularities on an open subset $D$ of $M$ if $u$ is a $C^{\infty}$ function on $D$ except a discrete subset $E$ of $D$ and for every $a \in D$ we can write

$$
|u(z)|=\left.|z|^{q} \prod_{i}|\log | g_{i}(z) v_{i}(z)\right|^{\mid t_{i}} u^{*}(z)
$$

around $a$ with real numbers $\sigma$ and $\tau_{i}$, positive $C^{\infty}$ functions $u^{*}, v_{i}$ and some nonzero holomorphic functions $g_{i}(z)$ satisfying the condition $g_{i}(0)=0$, where $z$ is a holomorphic local coordinate with $z(a)=0$ around $a$. We mean by a continuous function on $D$ with mild singularities a function with mild singularities which is complex-valued and continuous on the totality of $D$. 
Let $u$ be a function with mild singularities on $D$. We define a map $\nu_{u}$ of $D$ into $\mathbf{R}$ by

$$
\nu_{u}(a):=\text { the number } \sigma \text { with the property (4.2) for some } \tau \text { and } u^{*}
$$

for each $a \in D$, which does not depend on a choice of a holomorphic local coordinate $z$. By a divisor on $M$ we mean a map $\nu$ of $M$ into $\mathbf{R}$ such that the support $|\nu|:=\overline{\{p ; \nu(p) \neq 0\}}$ has no accumulation point in $M$. The above $\nu_{u}$ is a divisor on $D$, which we call the divisor of $u$. The divisor of a nonzero meromorphic function $\psi$ is nothing but the divisor $\nu_{\psi}$ stated in $\S 2$.

Definition 4.3. We call $d s^{2}$ a (conformal) pseudo-metric on $M$ if for each $a \in M$ we may write $d s^{2}=\lambda_{z}^{2}|d z|^{2}$ around $a$ with a nonnegative function $\lambda_{z}$ with mild singularities and a holomorphic local coordinate $z$. For a pseudo-metric $d z^{2}=\lambda_{z}^{2}|d z|^{2}$ we define the divisor of $d s^{2}$ by $\nu_{d s}=\nu_{\lambda_{z}}$. A continuous pseudo-metric $d s^{2}$ means a pseudo-metric such that $\lambda_{z}$ is continuous.

Derinition 4.4. For a pseudo-metric $d s^{2}=\lambda_{z}^{2}|d z|^{2}$ on $M$ we define the Ricci form by

$$
\operatorname{Ric}_{d s^{2}}=-d d^{c} \log \lambda_{z}^{2}
$$

as a current, which is well-defined on the totality of $M$ because it does not depend on the choice of holomorphic local coordinate $z$. We define also the total curvature $C(M)$ of $M$ by $C(M)=-\int_{M} \operatorname{Ric}_{d s^{2}}$ for a conformal metric $d s^{2}$ on $M$.

The Gaussian curvature $\mathscr{K}_{d s^{2}}$ of $d s^{2}$ is defined by

$$
\mathscr{K}_{d s^{2}}=-\frac{\Delta \log \lambda_{z}}{\lambda_{z}^{2}}=-\frac{4\left(\partial^{2} / \partial z \partial \bar{z}\right) \log \lambda_{z}}{\lambda_{z}^{2}}
$$

only on the set where $d s^{2} \neq 0$. The curvature $\mathscr{K}_{d s^{2}}$ is said to be strictly negative if there exists a positive constant $C$ such that $\operatorname{Ric}_{d s^{2}} \leq-C \Omega_{d s^{2}}$ on $M$ as currents, where $\Omega_{d s^{2}}$ denotes the area element with respect to the metric $d s^{2}$. As is well-known, if the universal covering surface of $M$ is biholomorphic with the unit disc in $\mathbf{C}$, then $M$ has the complete conformal metric with constant curvature -1 which is called the Poincaré metric of $M$ and denoted by $d \sigma_{M}^{2}$ in the following.

Let $\nu$ be a divisor on $M$. We denote by [ $\nu]$ the current which corresponds to $\nu$, namely the map of $\mathscr{D}$ into $\mathbf{C}$ defined by 


$$
[\nu](\varphi)=\int_{M} \nu \varphi=\sum_{z \in M} \nu(z) \varphi(z),
$$

where $\mathscr{D}$ denotes the space of all $C^{\infty}$ differentiable functions on $M$ with compact supports. In some cases, a $(1,1)$-form $\Omega$ on $M$ is regarded as a current on $M$ by defining $\Omega(\varphi):=\int_{M} \varphi \Omega$ for each $\varphi \in \mathscr{D}$.

For two $(1,1)$-currents $\Omega_{1}, \Omega_{2}$ on some open set $U$ and a positive constant $c$, by the notation $\Omega_{1} \prec_{c} \Omega_{2}$ we mean that there are divisor $\nu$ and a continuous real-valued bounded function $k$ with mild singularities on $U$ such that $\nu(z) \geq c$ for each $z \in|\nu|$ and

$$
\Omega_{1}+[\nu]=\Omega_{2}+d d^{c} \log k^{2}
$$

on $U$. For brevity, we mean by $\Omega_{1} \prec \Omega_{2}$ or $\Omega_{2} \succ \Omega_{1}$ that $\Omega_{1} \prec_{c} \Omega_{2}$ for some positive number $c$.

Obviously, we have the following:

Proposition 4.5. (i) If $\Omega_{1} \prec_{c} \Omega_{2}$ and $\Omega_{2} \prec_{c} \Omega_{3}$ for a constant $c>0$, then $\Omega_{1} \prec_{c} \Omega_{3}$.

(ii) If $\Omega_{1} \prec \Omega_{2}$, then $a \Omega_{1}+\Omega \prec a \Omega_{2}+\Omega$ for every $\Omega$ and $a>0$.

The purpose of this section is to prove the following:

THEOREM 4.6. Let $M$ be an open Riemann surface with a complete continuous pseudo-metric $d s^{2}$ and let $d \tau^{2}$ be a continuous pseudo-metric on $M$ whose curvature is strictly negative outside a compact set $K$. Assume that there exists a constant $p$ with $0<p<1$ such that

$$
-\operatorname{Ric}_{d s^{2}} \prec_{1-p} p\left(-\operatorname{Ric}_{d z^{2}}\right)
$$

on $M-K$. Then $M$ is of finite type, namely $M$ is biholomorphic with a compact Riemann surface from which finitely many points have been removed.

For the proof of Theorem 4.6, we recall the following generalization of Schwarz lemma.

LEMMA 4.7. Let $d \tau^{2}$ be a continuous pseudo-metric on $\Delta_{R}:=\{w ;|w|$ $<R\}$ whose curvature is strictly negative. Then, for some positive constant $C_{0}$,

$$
d \tau^{2} \leq C_{0} d \sigma_{\Delta_{R}}^{2}
$$

For the proof, see [1, pp. 12 14].

We need also the following result given by $\mathrm{A}$. Huber. 
Theorem 4.8. For an open Riemann surface $M$, if there is a complete conformal metric $d s^{2}$ on $M$ such that

$$
\int_{M} \max \left(-\mathscr{K}_{d s^{2}}, 0\right) \Omega_{d s^{2}}<+\infty
$$

then $M$ is of finite type, where $\Omega_{d^{2}}$ is the area element with respect to $d s^{2}$.

The proof is omitted. For the details, refer to [10, Theorem 13, p. 61, Theorem 15, p. 71], [16, Theorem 1, p. 316] and [3].

Proof of Theorem 4.6. By assumption, there are a divisor $\nu$ and a continuous real-valued bounded function $k$ on $M-K$ with mild singularities such that $\nu(z) \geq 1-p$ for every $z \in|\nu|$ and

$$
-\operatorname{Ric}_{d_{s^{2}}}+[\nu]=p\left(-\operatorname{Ric}_{d z^{2}}\right)+d d^{c} \log k^{2} .
$$

Here, we may assume that the divisor $\nu$ and the continuous function $k$ with mild singularities are defined on the totality of $M$ and $0 \leq k \leq 1$. Take a nowhere zero holomorphic 1-form $\omega$ and write

$$
d s^{2}=\lambda^{2}|\omega|^{2}, \quad d \tau^{2}=\eta^{2}|\omega|^{2},
$$

where $\lambda, \eta$ are continuous functions with mild singularities on $M$. Set

$$
u:=\frac{k n^{p}}{\lambda} \text {. }
$$

Then, $v:=\log u$ is harmonic on $M-(K \cup|\nu|), \nu_{u} \geq 1-p$ on $|\nu|-K$ and

$$
\lambda=e^{-v} k \eta^{p} \leq e^{-v} \eta^{p} .
$$

Define a new pseudo-metric

$$
d \rho^{2}:=e^{-2 v /(1-p)}|\omega|^{2}
$$

on $M$ and set $M_{1}:=\left\{a \in M ; \nu_{d \rho}(a) \geq 0\right\}$. Then, $d \rho^{2}$ is a metric on $M_{1}$ which is flat on $M_{1}-K$ and $\nu_{d \rho} \leq-1$ on $M-\left(K \cup M_{1}\right)$ because $\nu_{u} \geq 1-p$ on $\left|\nu_{u}\right|-K$. For the proof of Theorem 4.6, it suffices to prove that

(4.10) The surface $M_{1}$ is complete with respect to the metric $d \rho^{2}$.

In fact, if (4.10) is true, then $M_{1}$ is of finite type by Theorem 4.8, because

$$
\int_{M_{1}} \max \left(-\mathscr{K}_{d \rho^{2}}, 0\right) \Omega_{d \rho^{2}}=\int_{K} \max \left(-\mathscr{K}_{d \rho^{2}}, 0\right) \Omega_{d \rho^{2}}<\infty
$$


This implies that $M$ is also of finite type.

Assume that (4.10) is not true. Then

$$
d_{0}:=\operatorname{dist}_{d \rho}\left(K, \partial M_{1}\right)<+\infty,
$$

where $\operatorname{dist}_{d \rho}\left(K, \partial M_{1}\right)$ denotes the distance between $K$ and the boundary $\partial M_{1}$ of $M_{1}$. So, for a sufficiently small positive number $\delta$ there is a rectifiable curve $\gamma_{0}(t)(0 \leq t<1)$ such that $\gamma_{0}(0) \in K, \gamma_{0}(t)$ tends to $\partial M_{1}$ as $t \rightarrow 1$ and $L_{d \rho}\left(\gamma_{0}\right)<d_{0}+\delta$, where $L_{d \rho}\left(\gamma_{0}\right)$ denotes the length of the curve $\gamma_{0}$ with respect to the metric $d \rho^{2}$. If we take some $t_{0}$ sufficiently near to 1 , the point $p_{0}:=\gamma_{0}\left(t_{0}\right)$ satisfies the inequalities

$$
\operatorname{dist}_{d \rho}\left(K, p_{0}\right)>\frac{d_{0}}{2}, \quad L_{d \rho}\left(\gamma_{0} \mid\left[t_{0}, 1\right)\right)<\frac{d_{0}}{2},
$$

where $\gamma \mid[\alpha, \beta)$ denotes the part of $\gamma$ from $t=\alpha$ to $t=\beta$.

Since $d \rho^{2}$ is flat on $M_{1}-K$, there is an isometry $\Phi$ of a disc $\Delta_{R}:=$ $\{w \in \mathbf{C} ;|w|<R\}$ with the standard metric onto an open neighborhood of $p_{0}$ in $M_{1}-K$ with the metric $d \rho^{2}$ such that $\Phi(0)=p_{0}$. We take the largest $R(\leq+\infty)$ such that there is a local isometry $\Phi$ of $\Delta_{R}$ onto some open set in $M_{1}-K$ with $\Phi(0)=p_{0}$. Then, we see easily $R \leq L_{d \rho}\left(\gamma_{0} \mid\left[t_{0}, 1\right)\right)<d_{0} / 2$. By the definition of $R$, we can find a point $a_{0}$ in $\partial \Delta_{R}$ such that, for the line segment

$$
\Gamma: w=t a_{0} \quad(0 \leq t<1),
$$

the image $\gamma:=\Phi(\Gamma)$ tends to the boundary of $M_{1}-K$ as $t$ tends to 1 . Then, $\gamma$ cannot tend to the set $K$ nor $M-\left(K \cup M_{1}\right)$. In fact, if $\gamma$ tends to $K$, then we have an absurd conclusion

$$
R \geq \operatorname{dist}_{d \rho}\left(K, p_{0}\right)>d_{0} / 2
$$

and, if $\gamma$ tends to the set $M-\left(K \cup M_{1}\right)$, then $R=L_{d \rho}(\gamma)=+\infty$ because $\nu_{d \rho} \leq-1$ on $M-\left(K \cup M_{1}\right)$. Therefore, $\gamma$ tends to the boundary of $M$.

Now, we shall estimate the length of $\gamma$ with respect to the metric $d s^{2}$. To this end, we define a function $\tilde{\eta}$ with mild singularities by $d \tau=\tilde{\eta} d \rho$. Then, since

$$
d \tau=\eta|\omega|=\tilde{\eta} \mathrm{e}^{-v /(1-p)}|\omega|,
$$

we have

$$
\eta=\tilde{\eta} \mathrm{e}^{-v /(1-p)}
$$


By the use of (4.9), the length of $\gamma$ with respect to $d s^{2}$ is estimated by

$$
\begin{aligned}
L_{d s}(\gamma) & \leq \int_{r} e^{-v} \eta^{p}|\omega| \leq \int_{r} e^{-v} \tilde{\eta}^{p} e^{-p v /(1-p)}|\omega|=\int_{r} \tilde{\eta}^{p} d \rho \\
& =\int_{\Gamma}(\tilde{\eta} \cdot \Phi)^{p} \Phi^{*}(d \rho)=\int_{\Gamma}(\tilde{\eta} \cdot \Phi)^{p}|d w| .
\end{aligned}
$$

On the other hand, by the assumption of $d \tau$ the curvature of $\Phi^{*}(d \tau)$ is strictly negative on $\Delta_{R}$. It follows from Lemma 4.7 that

$$
\Phi^{*}(d \tau)=(\tilde{\eta} \cdot \Phi)|d w| \leqq C_{0} d \sigma_{\Delta R}
$$

for a positive constant $C_{0}$. Since the Poincaré metric $d \sigma_{\Delta R}^{2}$ is given by

$$
d \sigma_{\Delta_{R}}^{2}=C_{1}\left(\frac{R}{R^{2}-|w|^{2}}\right)^{2}|d w|^{2}
$$

for some positive constant $C_{1}$, we see

$$
L_{d s}(\gamma) \leq C_{2} \int_{\Gamma}\left(\frac{R}{R^{2}-|w|^{2}}\right)^{p}|d w| \leq \frac{C_{3}}{1-p} R^{1-p}<\infty,
$$

where each $C_{i}$ denotes some constant. This contradicts the completeness of $M$ with respect to $d s^{2}$. Therefore, we have (4.10) and so the proof of Theorem 4.6 is completed.

\section{§5. Modified defect relations}

Let $f$ be a nondegenerate holomorphic map of $M$ into $\mathbf{P}^{n}(\mathbf{C})$ and let

$$
H:(A, W)=0
$$

be a hyperplane in $\mathbf{P}^{n}(\mathbf{C})$, where $A$ is a unit vector. Take a reduced representation $f=\left(f_{0}: \cdots: f_{n}\right)$ on $M$ and set $F=\left(f_{0}, \cdots, f_{n}\right)$, where $f_{0}, \cdots, f_{n}$ are holomorphic functions without common zeros. We consider the divisor $\nu(f, H)=\nu_{F(H)}$ for the function $F(H):=(A, F)$. The $n$-truncated pull-back $f^{*}(H)^{[n]}$ of the divisor corresponding to $H$ stated in $\S 1$ is defined by

$$
f^{*}(H)^{[n]}=[\min (\nu(f, H), n)] .
$$

We have always

$$
f^{*}(H)^{[n]} \prec \Omega_{f} .
$$

In fact, if we take a nonzero holomorphic function $g$ with $\nu_{g}=\min (\nu(f, H), n)$, for $k:=|F(H)| /|| f \|$ and $u:=|F(H) / g|$ we see $0 \leq k \leq 1$ and 


$$
f^{*}(H)^{[n]}+d d^{c} \log u^{2}=d d^{c} \log \|f\|^{2}+d d^{c} \log k^{2} .
$$

As $d d^{c} \log u^{2}=\left[\nu_{u}\right]$ and $\nu_{u} \geq 1$ on $\left|\nu_{u}\right|$ by Poincaré-Lelong formula, we get (5.1).

Definition 5.2. For an arbitrary compact subset $K$ of $M$ we set

$$
\eta_{f}(H)^{K}:=\inf \left\{\eta>0 ; f^{*}(H)^{[n]} \prec \eta \Omega_{f} \text { on } M-K\right\} .
$$

We define the modified $H$-defect of $H$ for $f$ by

$D_{f}(H):=1-\inf \left\{\eta_{f}(H)^{K} ; K\right.$ is an arbitrary compact subset of $\left.M\right\}$.

Remark 5.3. The notion of $\mathrm{H}$-defect defined in the previous paper [8] is essentially equal to the quantity $\eta_{f}(H)^{K}$ for the case where $K=\varnothing$.

The modified $\mathrm{H}$-defect has the following properties.

Proposition 5.4. (i) $0 \leq D_{f}(H) \leq 1$.

(ii) If there exists a bounded nonzero holomorphic function $\mathrm{g}$ on $M-K$ for a compact set $K$ such that $\nu_{g} \geq \min (\nu(f, H), n)$, or particularly, if $\# f^{-1}(H)$ is finite, then $D_{f}(H)=1$.

(iii) If $\nu(f, H)(a) \geq m$ at every $a \in f^{-1}(H)-K$ for some compact set $K$, then $D_{f}(H) \geq 1-n / m$.

Proof. The assertion (i) is obvious. The function $k:=|g|$ is a bounded continuous function with mild singularities. Since

$$
f^{*}(H)^{[n]}+\left[\nu_{g}-\min (\nu(f, H), n)\right]=\eta \Omega_{f}+d d^{c} \log k^{2}
$$

on $M-K$ for $\eta=0$, we have (ii). To see (iii), consider the bounded function

$$
k:=\left(\frac{|F(H)|}{\|f\|}\right)^{n / m}
$$

By the assumption, $k$ is a continuous function with mild singularities and satisfies

$$
f^{*}(H)^{[n]}+\left[\frac{n}{m} \nu(f, H)-\min (\nu(f, H), n)\right]=\frac{n}{m} d d^{c} \log \|f\|^{2}+d d^{c} \log k^{2}
$$

on $M-K$. Since $(n / m) \nu(f, H) \geq n \geq \min (\nu(f, H), n)$ on $f^{-1}(H)-K$ by the assumption, we have $D_{f}(H) \geq 1-n / m$.

Now, we recall the classical defect for a nondegenerate holomorphic map of an open neighborhood of $\Delta_{R, \infty}:=\{z ; R \leq|z|<+\infty\}$ into $\mathbf{P}^{n}(\mathbf{C})$. 
The order function of $f$ and the counting function (truncated by $n$ ) of a hyperplane $H$ for $f$ are defined by

$$
\begin{gathered}
T_{f}(r)=\int_{R}^{r} \frac{d t}{t} \int_{R \leq|z|<t} \Omega_{f} \quad(R<r<+\infty), \\
N_{f}(r)^{[n]}=\int_{R}^{r} \frac{d t}{t} \int_{R \leq|z|<t} f^{*}(H)^{[n]} \quad(R<r<+\infty)
\end{gathered}
$$

respectively. The classical defect (truncated by $n$ ) is defined by

$$
\delta_{f}(H)^{[n]}=1-\limsup _{r \rightarrow \infty} \frac{N_{f}(r)^{[n]}}{T_{f}(r)} .
$$

We can easily prove the following relation by the same arguments as in the previous paper $[7, \S 1]$.

Proposition 5.5. Let $f$ be a nondegenerate holomorphic map of an open Riemann surface $M$ into $\mathbf{P}^{n}(\mathbf{C})$. Assume that, for some open subset $G$ of $M$ with compact boundary $\partial G$, there is a biholomorphic map $\Phi$ of a neighborhood of $M-G$ onto a neighborhood of $\Delta_{R, \infty}$ such that $\Phi(M-G)=$ $\Delta_{R, \infty}$ and $\Phi(\partial G)=\{z ;|z|=R\}$, and assume that the restriction $\tilde{f}:=f \cdot \Phi^{-1} \mid \Delta_{R, \infty}$ has an essential singularity at $\infty$, namely, the map $\tilde{f}(1 / z):\{z ; 0<|z|<1 / R\}$ $\rightarrow \mathbf{P}^{n}(\mathbf{C})$ has no holomorphic extension to $\{z ;|z|<1 / R\}$. Then, for every hyperplane $H$

$$
0 \leq D_{f}(H) \leq \delta_{\tilde{f}}(H)^{[n]} \leq 1
$$

The classical defect relation improved by Nochka is given as follows:

THEOREM 5.6. Let $f$ be a nondegenerate holomorphic map of an open neighborhood of $\Delta_{R, \infty}$ into $\mathbf{P}^{n}(\mathbf{C})$ such that it has an essential singularity at $\infty$. Take $q(>2 N-n+1)$ hyperplanes $H_{j}(1 \leq j \leq q)$ in $\mathbf{P}^{n}(\mathbf{C})$ located in $N$-subgeneral position and consider Nochka constants $\omega(j)$. Then,

$$
\sum_{j=1}^{q} \omega(j) \delta_{f}\left(H_{j}\right)^{[n]} \leq n+1 .
$$

For the proof, see [12] or [2].

Now, we consider a nonconstant holomorphic map $f$ of an open Riemann surface $M$ with a pseudo-metric $d s^{2}$ into $\mathbf{P}^{n}(\mathbf{C})$.

Definition 5.7. For an arbitrary compact set $K$ we set

$$
\rho_{f}^{K}:=\inf \left\{\rho>0 ;-\operatorname{Ric}_{d s^{2}} \prec \rho \Omega_{f} \text { on } M-K\right\},
$$


where we set $\rho_{f}^{K}=+\infty$ if there is no $\rho$ with the above property. We define the $H$-order of $f$ by

$$
\rho_{f}=\inf \left\{\rho_{f}^{K} ; K \text { is an arbitrary compact subset of } M\right\} .
$$

To state the modified defect relation, we give another definition.

Definition 5.8. Let $M$ be an open Riemann surface of finite type, namely $M$ is biholomorphic with a compact Riemann surface $\bar{M}$ with finitely many points removed. A holomorphic map of $M$ into $\mathbf{P}^{n}(\mathbf{C})$ is said to be transcendental if $f$ has no holomorphic extension to $\bar{M}$.

THEOREM 5.9. Let $M$ be an open Riemann surface with a complete continuous pseudo-metric $d s^{2}$ and let $f$ be a nondegenerate holomorphic map of $M$ into $\mathbf{P}^{n}(\mathbf{C})$. Take $q(>2 N-n+1)$ hyperplanes in $N$-subgeneral position and Nochka constants $\omega(j)(1 \leq j \leq q)$. If $M$ is not of finite type or else $f$ is transcendental, then

$$
\sum_{j=1}^{q} \omega(j) D_{f}\left(H_{j}\right) \leq n+1+\frac{\rho_{f} n(n+1)}{2} .
$$

Before proving Theorem 5.9, we notice that Theorem 1.1 stated in $\S 1$ is an easy consequence of Theorem 5.9. Let $A$ denote the right hand side of (1.2). Then we have

$$
\begin{aligned}
A & \geq 2 N-n+1+\frac{\rho_{f} \sigma_{n}}{\theta}=q+\frac{1}{\theta}\left(n+1+\rho_{f} \sigma_{n}-\sum_{j=1}^{q} \omega(j)\right) \\
& \geq q+\sum_{j=1}^{q} \frac{\omega(j)}{\theta}\left(D_{f}\left(H_{j}\right)-1\right) \geq q+\sum_{j=1}^{q}\left(D_{f}\left(H_{j}\right)-1\right)=\sum_{j=1}^{q} D_{f}\left(H_{j}\right)
\end{aligned}
$$

by the help of Theorem 2.2, where $\sigma_{n}:=n(n+1) / 2$. This gives Theorem 1.1 .

Proof of Theorem 5.9. It suffices to prove (5.10) for the case $\rho_{f}<+\infty$. Moreover, we may assume that $M$ is not of finite type. In fact, if $M$ is of finite type and $f$ is transcendental, Theorem 5.9 is obvious from Theorem 5.6 .

Take arbitrary constants $\rho>0$ and $\eta_{j}(1 \leq j \leq q)$ satisfying the condition

$$
-\operatorname{Ric}_{d s^{2}} \prec \rho \Omega_{f}, \quad f^{*}\left(H_{j}\right)^{[n]} \prec \eta_{j} \Omega_{f}
$$

on $M-K$ for a compact set $K$. By definition, there are divisors $\left[\nu_{j}\right]$ and bounded continuous real-valued functions $k_{j}$ with mild singularities such that $\nu_{j} \geq c_{j}$ on $\left|\nu_{j}\right|$ for some positive constant $c_{j}$ and 


$$
f^{*}\left(H_{j}\right)^{[n]}+\left[\nu_{j}\right]=\eta_{j} \Omega_{f}+d d^{c} \log k_{j}^{2}
$$

on $M-K$. Here, we may assume that $0 \leq k_{j} \leq 1$. Set $h_{j}:=k_{j}\|f\|^{\eta_{j}}$. Then, each $\log h_{j}$ is harmonic on $M-\left(K \cup\left|\nu_{h_{j}}\right| \cup f^{-1}\left(H_{j}\right)\right)$ and $\nu_{h_{j}}-\min \left(\nu\left(f, H_{j}\right), n\right)$ $=\nu_{j}$.

Set

$$
\gamma:=\theta(q-2 N+n-1)-\sum_{j=1}^{q} \omega(j) \eta_{j}
$$

We shall prove

$$
\gamma \leq \rho \sigma_{n}
$$

Assume that $\gamma>\rho \sigma_{n}$. Take a positive number $\varepsilon$ with $\gamma>\varepsilon \sigma_{n+1}$ and set

$$
p:=\frac{\rho\left(\sigma_{n}+\tau_{n} \varepsilon\right)}{\gamma-\varepsilon \sigma_{n+1}} .
$$

For an arbitrary holomorphic local coordinate $z$, using the same notation as in $\S 3$, we set

$$
\eta_{z}:=\left(\frac{\|f\|^{\gamma-\varepsilon \sigma_{n+1}}\left|F_{n}\right| \prod_{j=1}^{q}\left|h_{j}\right|^{\omega(j)} \prod_{k=0}^{n}\left|F_{k}\right|^{\varepsilon}}{\prod_{j=1}^{q}\left(\left|F\left(H_{j}\right)\right| \prod_{k=0}^{n-1} \log \left(a / \varphi_{k}\left(H_{j}\right)\right)\right)^{\omega(j)}}\right)^{1 /\left(\sigma_{n}+\varepsilon \tau_{n}\right)} .
$$

Then, the pseudo-metric $d \tau^{2}:=\eta_{z}^{2}|d z|^{2}$ is defined on $M-K$ independently of the choice of holomorphic local coordinate $z$. Here, $\eta_{z}=0$ if $\varphi_{k}\left(H_{j}\right)$ $=0$. On the other hand, if we set

$$
\varphi:=\frac{\left|F_{n}\right|}{\prod_{j=1}^{q}\left|F\left(H_{j}\right)\right|^{(j)}}
$$

and $\nu_{0}:=\nu_{\varphi}+\sum_{j=1}^{q} \omega(j) \nu_{h_{j}}$, then $\nu_{0} \geq c^{\prime}$ on $\left|\nu_{0}\right|$ for some $c^{\prime}>0$, because

$$
\begin{aligned}
\nu_{0} & =\sum_{j=1}^{q} \omega(j)\left(\nu_{h_{j}}-\min \left(\nu\left(f, H_{j}\right), n\right)\right)+\left(\nu_{\varphi}+\sum_{j=1}^{q} \omega(j) \min \left(\nu\left(f, H_{j}\right), n\right)\right) \\
& \geq \sum_{j=1}^{q} \omega(j)\left(\nu_{h_{j}}-\min \left(\nu\left(f, H_{j}\right), n\right)\right)
\end{aligned}
$$

by Lemma 2.4. This implies that $d \tau^{2}$ is a continuous pseudo-metric on $M-K$. We suitably extend $d \tau^{2}$ to a continuous pseudo-metric on the totality of $M$.

We shall next prove that $d \tau^{2}$ has strictly negative curvature on $M-K$. To this end, we recall the following inequality given in [8, Proposition 3.8]: 


$$
d d^{c} \log \left|F_{0}\right|^{2}\left|F_{1}\right|^{2} \cdots\left|F_{n-1}\right|^{2} \geq \frac{\tau_{n}}{\sigma_{n}}\left(\frac{\left|F_{0}\right|^{2} \cdots\left|F_{n}\right|^{2}}{\left|F_{0}\right|^{2 \sigma_{n+1}}}\right)^{1 / z_{n}} \sqrt{-1} d z \wedge d \bar{z}
$$

This and Theorem 3.4 yield that

$$
\begin{aligned}
& d d^{c} \log \eta_{z}^{2} \geq \frac{\gamma-\varepsilon \sigma_{n+1}}{\sigma_{n}+\varepsilon \tau_{n}} \Omega_{f}+\frac{\varepsilon}{2\left(\sigma_{n}+\varepsilon \tau_{n}\right)} d d^{c} \log \left(\left|F_{0}\right|^{2} \cdots\left|F_{n-1}\right|^{2}\right) \\
& +\frac{1}{2\left(\sigma_{n}+\varepsilon \tau_{n}\right)} d d^{c} \log \frac{\prod_{\substack{n-1 \\
k=0}}^{n-\left.F_{k}\right|^{2 \varepsilon}}}{\prod_{1 \leq j \leq q, 0 \leq k \leq n-1} \log ^{2 \omega(j)}\left(a / \varphi_{k}\left(H_{j}\right)\right)} \\
& \geq \frac{\varepsilon}{2} \frac{\tau_{n}}{\sigma_{n}\left(\sigma_{n}+\varepsilon \tau_{n}\right)}\left(\frac{\left|F_{0}\right|^{2} \cdots\left|F_{n}\right|^{2}}{\left|F_{0}\right|^{2 \sigma_{n+1}}}\right)^{1 / \tau_{n}} d z \wedge d^{c} z \\
& +C_{0}\left(\frac{\left|F_{0}\right|^{2 \theta(q-2 N+n-1)}\left|F_{n}\right|^{2}}{\prod_{j=1}^{q}\left(\left|F\left(H_{j}\right)\right|^{2} \prod_{\left.\substack{n-1 \\
k=0} \log ^{2}\left(a / \varphi_{k}\left(H_{j}\right)\right)\right)^{\omega(j)}}\right.}\right)^{1 / \sigma_{n}} \sqrt{-1} d z \wedge d \bar{z} .
\end{aligned}
$$

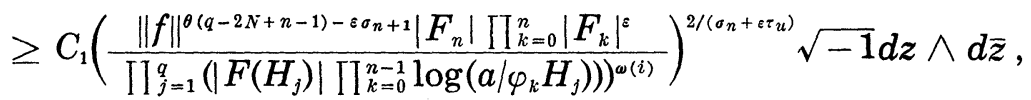

where $C_{0}, C_{1}$ are some positive constants. On the other hand, since $\left|h_{j}\right|$ $\leq\|f\|^{\eta_{j}}$

$$
\|f\|^{\theta(q-2 N+n-1)-\varepsilon \sigma_{n+1}} \geq\|f\|^{r-\varepsilon \sigma_{n+1}} h_{1}^{\omega(1)} \cdots h_{q}^{\omega(q)}
$$

on $M-K$. This concludes that $d d^{c} \log \eta^{2} \geq C_{2} \eta^{2}$ for some positive constant $C_{2}$. Therefore, $d \tau^{2}$ has strictly negative curvature on $M-K$.

Now, we represent each hyperplane $H_{j}(1 \leq j \leq q)$ as

$$
H_{j}: a_{j 0} w_{0}+\cdots+a_{j n} w_{n}=0 .
$$

For some holomorphic local coordinate $z$ and each pair of indices $j, k$, we choose indices $i_{1}, \cdots, i_{k}$ with $1 \leq i_{1}<\cdots<i_{k} \leq q$ such that

$$
\psi_{k j}^{z}:=\sum_{l \neq i_{1}, \cdots, i_{k}} a_{j l} W\left(f_{l}, f_{i_{1}}, \cdots, f_{i_{k}}\right) \not \equiv 0 .
$$

For convenience' sake, we set $\psi_{j 0}^{z}=F\left(H_{j}\right)$. By the theorem of identity, $\psi_{j k}^{z} \not \equiv 0$ for every holomorphic local coordinate $z$. We now define

$$
k:=\left(\frac{\prod_{1 \leq j \leq q, 0 \leq k \leq n-1}\left|\psi_{j k}^{z}\right|^{\varepsilon / q} \log ^{\omega(j)}\left(a / \varphi_{k}\left(H_{j}\right)\right)}{\prod_{0 \leq k \leq n-1}\left|F_{k}\right|^{\varepsilon}}\right)^{1 /\left(\sigma_{n}+\varepsilon \tau_{n}\right)}
$$

Then, $k$ is bounded because

$$
\begin{aligned}
\frac{\left|\psi_{j k}^{z}\right|^{\varepsilon / q} \log ^{\omega(j)}\left(a / \varphi_{k}\left(H_{j}\right)\right)}{\left|F_{k}\right|^{\varepsilon / q}} & \leq\left(\frac{\left|F_{k}\left(H_{j}\right)\right|}{\left|F_{k}\right|}\right)^{\varepsilon / q} \log ^{\omega(j)}\left(a / \varphi_{k}\left(H_{j}\right)\right) \\
& \leq \sup _{0<x \leq 1} x^{\varepsilon / q} \log ^{\omega(j)}\left(\frac{a}{x}\right)<+\infty .
\end{aligned}
$$


Set

$$
v_{1}:=|\varphi| \prod_{j=1}^{q}\left|h_{j}\right|^{(j)}, \quad v_{2}:=\prod_{1 \leq j \leq q, 0 \leq k \leq n-1}\left|\psi_{j k}^{z}\right|^{\varepsilon / q}, v:=v_{1} v_{2} .
$$

Then $\log v$ is harmonic on $M-\left(K \cup\left|\nu_{v}\right|\right), \nu_{v_{1}} \geq c^{\prime \prime}$ on $\left|\nu_{v_{1}}\right|$ for some $c^{\prime \prime}$, not depending on each $\varepsilon$, and $\nu_{v_{2}} \geq \varepsilon / q$ on $\left|\nu_{v_{2}}\right|$. We consider the function $\eta_{z}$ for some $\varepsilon$ such that

$$
\frac{\gamma-\rho \sigma_{n}}{\sigma_{n+1}+\rho \tau_{n}}>\varepsilon>\max \left(\frac{\gamma-\rho \sigma_{n}}{\rho / q+\sigma_{n+1}+\rho \tau_{n}}, \frac{\gamma-\rho \sigma_{n}-c^{\prime \prime} \rho}{\sigma_{n+1}+\rho \tau_{n}}\right) .
$$

and, moreover, $-\operatorname{Ric}_{d_{s^{2}}} \prec_{1-p} \rho \Omega_{f}$. Then, we have $0<p<1$ and

$$
\nu_{v} \geq \min \left(\frac{\varepsilon}{q}, c^{\prime \prime}\right) \geq \frac{\left(\gamma-\varepsilon \sigma_{n-1}\right)(1-p)}{\rho}
$$

on $\left|\nu_{v}\right|-K$. Since

$$
k \eta_{z}=\|f\|^{\left(\gamma-\varepsilon \sigma_{n}+1\right) /\left(\sigma_{n}+\varepsilon \tau_{n}\right)} V^{1 /\left(\sigma_{n}+\varepsilon \tau_{n}\right)},
$$

we have

$$
\rho \Omega_{f}+\left[\frac{\rho}{\gamma-\varepsilon \sigma_{n+1}} \nu_{v}\right]=p d d^{c} \log \eta_{z}^{2}+d d^{c} \log k^{2 p} .
$$

This gives that

$$
-\operatorname{Ric}_{d^{2}} \prec_{1-p} p\left(-\operatorname{Ric}_{d \tau^{2}}\right)
$$

on $M-K$. This contradicts the assumption by Theorem 4.6. Thus, we conclude (5.12).

By Theorem 2.2, we can rewrite (5.12) as

$$
\sum_{j=1}^{q} \omega(j)\left(1-\eta_{j}\right) \leq n+1+\rho \sigma_{n} .
$$

Taking the supremum of the left hand side and the infimum of the right hand side for all admissible constants $\eta_{j}, \rho$ and compact set $K$, we can conclude

$$
\sum_{j=1}^{q} \omega(j) D_{f}\left(H_{j}\right) \leq n+1+\rho_{f} \sigma_{n} .
$$

The proof of Theorem 5.9 is completed. 


\section{§ 6. Modified defect relations for Gauss maps}

Let $x=\left(x_{1}, \cdots, x_{m}\right): M \rightarrow \mathbf{R}^{m}$ be a (possibly branched) minimal surface. By $S$ we denote the set of all branch points, namely points where the Jacobi matrix of $x$ are not of maximal rank. The set of all oriented 2-planes in $\mathbf{R}^{m}$ may be identified with the quadric $Q_{m-2}(\mathbf{C})$ in $\mathbf{P}^{m-1}(\mathbf{C})$. By definition, the Gauss map $G$ of $M$ is the map of $M-S$ into $Q_{m-2}(\mathbf{C})$ which maps each $p \in M-S$ to the point in $Q_{m-2}(\mathbf{C})$ corresponding to the oriented tangent plane of $M$ at $p$. The surface $M$ is considered an open Riemann surface with the conformal pseudo-metric $d s^{2}$ induced from the standard metric on $\mathbf{R}^{m}$. Taking a holomorphic local coordinate $z$, we set $f_{i}:=\partial x_{i} / \partial z$, which are holomorphic by the assumption of $M$. The set $S$ coincides with the set of common zeros of the functions $f_{i}(1 \leq i \leq m)$.

Definition 6.1. We now define the branching divisor of $M$ by

$$
\nu_{d s}=\min \left\{\nu_{f_{i}} ; 1 \leq i \leq m\right\},
$$

which is well-defined on the totality of $M$ independently of holomorphic local coordinate $z$.

The Gauss map $G$ is locally given by

$$
G=\left(f_{1}: \cdots: f_{m}\right)
$$

on $M-S$. Take a nonzero holomorphic function $h$ on $M$ with $\nu_{h}=\nu_{d s}$. If we set $g_{i}:=f_{i} / h(1 \leq i \leq m)$, we have

$$
G=\left(g_{1}: g_{2}: \cdots: g_{m}\right)
$$

on the common domain of $g_{i}$ 's except $S$. The right hand side of this has the holomorphic extension across $S$. We define the Gauss map $G$ of $M$ to be the holomorphic map of $M$ into $\mathbf{P}^{m-1}(\mathbf{C})$ locally given by the right hand side of the above identity.

Definition 6.2. For an arbitrary compact subset $K$ of $M$ we set

$$
\rho_{d s}^{K}:=\inf \left\{\rho ;\left[\nu_{d s}\right] \prec \rho \Omega_{G} \text { on } M-K\right\} .
$$

We define the branching $H$-order of $M$ by

$$
\rho_{d s}:=\inf \left\{\rho_{d s}^{K} ; K \text { is an arbitrary compact subset of } M\right\} .
$$

Obviously, if $x: M \rightarrow \mathbf{R}^{n}$ is an immersion, then $\rho_{d s}=0$.

Theorem 6.3. Let $x: M \rightarrow \mathbf{R}^{m}$ be a complete minimal surface and 
$G: M \rightarrow \mathbf{P}^{N}(\mathbf{C})$ the Gauss map of $M$, where $N=m-1$. Consider the smallest projective linear subspace $\mathbf{P}^{n}(\mathbf{C})$ of $\mathbf{P}^{N}(\mathbf{C})$ which includes $G(M)$. Take $q$ hyperplanes $H_{1}, \cdots, H_{q}$ in $\mathbf{P}^{N}(\mathbf{C})$ such that $\tilde{H}_{j}:=H_{j} \cap \mathbf{P}^{n}(\mathbf{C})(1 \leq j \leq q)$ are hyperplanes in $\mathbf{P}^{n}(\mathbf{C})$ located in $N$-subgeneral position, where $q>2 N$ $-n+1$. If $M$ is not of finite type, then for Nochka weight $\omega(j)$ for $\tilde{H}_{j}$

$$
\sum_{j=1}^{q} \omega(j) D_{G}\left(\tilde{H}_{j}\right) \leq n+1+\frac{\left(1+\rho_{d s}\right) n(n+1)}{2} .
$$

Proof. By assumption, the Gauss map $G$ is nondegenerate as the map into $\mathbf{P}^{n}(\mathbf{C})$. Take a system of homogeneous coordinate $\left(w_{0}: \cdots: w_{N}\right)$ on $\mathbf{P}^{N}(\mathbf{C})$ such that $\mathbf{P}^{n}(\mathbf{C}):=\left\{\left(w_{0}: \cdots: w_{N}\right) ; w_{n+1}=\cdots=w_{N}=0\right\}$ and a reduced representation $G=\left(g_{0}: \cdots: g_{n}\right)$ of $G$ as a map into $\mathbf{P}^{n}(\mathbf{C})$. So, we have

$$
d s^{2}=2\|f\|^{2}|d z|^{2} \leq C|h|^{2}\|g\|^{2}|d z|^{2}
$$

for a constant $C>0$ and $\|g\|:=\left(\left|g_{0}\right|^{2}+\cdots+\left|g_{n}\right|^{2}\right)^{1 / 2}$. Then, $f_{i}$ are linear combinations of $h g_{0}, \cdots, h g_{n}$ for a holomorphic function $h$ with $\nu_{h}=\nu_{d s}$. So, for each $\rho \geq 0$ such that $\left[\nu_{d s}\right] \prec \rho \Omega_{G}$ on $M$ outside a compact set $K$, we have

$$
-\operatorname{Ric}_{d s^{2}} \prec(\rho+1) \Omega_{G} \text {. }
$$

Taking the infimum of the right hand side for various $\rho$, we obtain

$$
\rho_{i} \leq \rho_{d s}+1
$$

Theorem 6.3 is now immediate consequence of Theorem 5.9.

Corollary 6.5. Let $x: M \rightarrow \mathbf{R}^{m}$ be a complete nonflat minimal surface with infinite total curvature, let $G: M \rightarrow \mathbf{P}^{N}(\mathbf{C})$ be the Gauss map of $M$ and let $n$ be the least dimension of projective linear subspaces of $\mathbf{P}^{N}(\mathbf{C})$ which include $G(M)$, where $N=m-1$. Then, for arbitrary hyperplanes $H_{1}, \cdots$, $H_{q}$ in $\mathbf{P}^{N}(\mathbf{C})$ located in general position and not including $G(M)$,

$$
\sum_{j=1}^{q} D_{G}\left(H_{j}\right) \leq 2 N-n+1+\frac{\left(1+\rho_{d s}\right) n(2 N-n+1)}{2} .
$$

Proof. Let us denote by $\mathbf{P}^{n}(\mathbf{C})$ the smallest projective linear subspace which includes $G(M)$. Then, as is easily seen, hyperplanes $H_{1} \cap \mathbf{P}^{n}(\mathbf{C})$, $\cdots, H_{q} \cap \mathbf{P}^{n}(\mathbf{C})$ of $\mathbf{P}^{n}(\mathbf{C})$ are located in $N$-subgeneral position. If $M$ is biholomorphic with a compact Riemann surface $\bar{M}$ with finitely many points removed and $G$ is not transcendental, then $\operatorname{Ric}_{d_{s}^{2}}$ is continuously 
extended to $\bar{M}$ and so $C(M)=C(\bar{M})<+\infty$, which contradicts the assumption. So, we have the situation in Theorem 1.1 for the map $G$ considered as a map into $\mathbf{P}^{n}(\mathbf{C})$. Since $\rho_{G} \leq \rho_{d s}+1$ by (6.4), Corollary 6.5 is a direct result of Theorem 1.1.

We shall give here the proof of Theorem 1.3. By assumption the Gauss map $G$ is not a constant. We have (6.6) for some $n$ with $1 \leq n \leq N$. Here, $\rho_{d s}=0$ because $M$ has no branch points. These imply that

$$
\begin{aligned}
\sum_{j=1}^{q} D_{G}\left(H_{j}\right) & \leq 2 N-n+1+\frac{n(2 N-n+1)}{2} \\
& =\frac{(N+1)(N+2)-(N-n)(N-n-1)}{2} \\
& \leq \frac{(N+1)(N+2)}{2}=\frac{m(m+1)}{2} .
\end{aligned}
$$

This gives Theorem 1.3.

Now, we consider a holomorphic curve in $\mathbf{C}^{m}$ given by a nonconstant holomorphic map $w=\left(w_{1}, w_{2}, \cdots, w_{m}\right): M \rightarrow \mathbf{C}^{m}$. The space $\mathbf{C}^{m}$ is identified with $\mathbf{R}^{2 m}$ by associating a point $\left(x_{1}+\sqrt{-1} y_{1}, \cdots, x_{m}+\sqrt{-1} y_{m}\right) \in \mathbf{C}^{m}$ with $\left(x_{1}, y_{1}, \cdots, x_{m}, y_{m}\right)$. The curve $w: M \rightarrow \mathbf{C}^{m}$ is considered as a minimal surface $w=\left(x_{1}, y_{1}, \cdots, x_{m}, y_{m}\right): M \rightarrow \mathbf{R}^{2 m}$. By Cauchy-Riemann's equations, we know

$$
f_{i}:=\frac{\partial x_{i}}{\partial z}=\sqrt{-1} \frac{\partial y_{i}}{\partial z} \quad(1 \leq i \leq m)
$$

Therefore, the Gauss map of $M$ is given by

$$
G=\left(f_{1}:-\sqrt{-1} f_{1}: \cdots: f_{m}:-\sqrt{-1} f_{m}\right)
$$

and so $G(M)$ is included in the projective linear subspace

$$
\mathbf{P}^{m-1}(\mathbf{C}):=\left\{\left(u_{1}: v_{1}: \cdots: u_{m}: v_{m}\right) \in \mathbf{P}^{2 m-1}(\mathbf{C}) ; u_{i}=\sqrt{-1} v_{i}(1 \leq i \leq m)\right\} .
$$

Particularly, if $M$ is not included in any proper affine subspace of $\mathbf{C}^{m}, G$ is nondegenerate as a map into $\mathbf{P}^{m-1}(\mathbf{C})$. The Gauss map $G$ considered as a map of $M$ into $\mathbf{P}^{m-1}(\mathbf{C})$ is the same as the complex Gauss map of $M$ defined in the previous paper [8, p. 369].

As an easy consequence of Theorem 6.3, we have the following improvement of [8, Theorem 2.10].

Corollary 6.7. Let $w: M \rightarrow \mathbf{C}^{m}$ be a holomorphic curve in $\mathbf{C}^{m}$ which 
is complete and not included in any affine hyperplane, and let $G$ be the Gauss map of $M$ considered as a map of $M$ into the above-mentioned space $\mathbf{P}^{n-1}(\mathbf{C})$. If $M$ is not of finite type, then

$$
\sum_{j=1}^{q} D_{G}\left(H_{\jmath}\right) \leq m+\frac{\left(\rho_{d s}+1\right) m(m-1)}{2}
$$

for arbitrary hyperplanes $H_{1}, \cdots, H_{q}$ in $\mathbf{P}^{n-1}(\mathbf{C})$ in general position.

We next consider a minimal surface $x=\left(x_{1}, x_{2}, x_{3}\right): M \rightarrow \mathbf{R}^{3}$. In this case, the quadric $Q_{1}(\mathbf{C})$ is canonically biholomorphic with $\mathbf{P}^{1}(\mathbf{C})$. Instead of the Gauss map $G: M \rightarrow Q_{1}(\mathbf{C})$ we may study the classical Gauss map $g: M \rightarrow \mathbf{P}^{1}(\mathbf{C})$ defined by

$$
g=\left(f_{3}: f_{1}-\sqrt{-1} f_{2}\right),
$$

where $f_{i}=\partial x_{i} / \partial z(i=1,2,3)$. Then, the metric of $M$ is given by

$$
d s^{2}=|h|^{2}\left(\left|g_{0}\right|^{2}+\left|g_{1}\right|^{2}\right)^{2}|d z|^{2}
$$

for a reduced representation $g=\left(g_{0}: g_{1}\right)$ and a nonzero holomorphic function $h$ with $\nu_{h}=\min \left(\nu_{1}, \nu_{2}, \nu_{3}\right)\left(\right.$ cf., $\left[7\right.$, p. 254]). Since $\nu_{d s}=\nu_{h}$, we have $-\operatorname{Ric}_{d s} \prec(\rho+2) \Omega_{g}$ whenever $\left[\nu_{d s}\right] \prec \rho \Omega_{g}$. This yields

$$
\rho_{g} \leq \rho_{d s}+2 \text {. }
$$

According to Theorem 6.9, we can easily prove the following:

Theorem 6.8. Let $x: M \rightarrow \mathbf{R}^{3}$ be a nonflat complete minimal surface which has infinite total curvature, and let $g: M \rightarrow \mathbf{P}^{1}(\mathbf{C})$ be the classical Gauss map. Then, for arbitrary distinct points $\alpha_{1}, \cdots, \alpha_{q}$ in $\mathbf{P}^{1}(\mathbf{C})$,

$$
\sum_{j=1}^{q} D_{g}\left(\alpha_{j}\right) \leq 4+\rho_{d s} .
$$

EXAMPLE 6.10. We give here an example of a minimal surface with infinite total curvature, for which the branching H-order is equal to two and the equality holds for the above modified defect relation (6.9).

Our construction is due to the Weierstrass-Enneper representation theorem for minimal surfaces in $\mathbf{R}^{3}$. We shall suitably choose meromorphic functions $f$ and $g$ on a simply connected open Riemann surface $\tilde{M}$ and show that, for the meromorphic 1 -forms

$$
\varphi_{1}:=\frac{\left(1-g^{2}\right) f d z}{2}, \quad \varphi_{2}:=\sqrt{-1} \frac{\left(1+g^{2}\right) f d z}{2}, \quad \varphi_{3}:=g f d z
$$


and a point $z_{0} \in \tilde{M}$, the map

$$
\left(x_{1}, x_{2}, x_{3}\right):=\left(\operatorname{Re} \int_{z_{0}}^{\zeta} \varphi_{1}(z), \operatorname{Re} \int_{z_{0}}^{\zeta} \varphi_{2}(z), \operatorname{Re} \int_{z_{0}}^{\zeta} \varphi_{3}(z)\right)
$$

of $\tilde{M}$ into $\mathbf{R}^{3}$ gives a minimal surface with the desired properties. To this end, we first take a meromorphic function $\phi$ on $\mathbf{C}$ such that $\phi$ takes the values $a_{i}(1 \leq i \leq 3)$ with multiplicity three and all zeros of $\phi(z)-w$ are simple for every $w \in \mathbf{C}-\left\{a_{1}, a_{2}\right\}$, where $a_{3}=\{\infty\}$. For the existence of such a function, see [9, p. 45]. Consider the domain $D:=\mathbf{C}-\left(\phi^{-1}\left(a_{1}\right) \cup \phi^{-1}\left(a_{2}\right)\right)$ and the analytic subset $V^{*}:=\left\{(z, w) ; \phi(z)=w^{3}\right\}$ of $D \times \mathbf{C}$. Let $\hat{M}$ be the normalization of the closure of $V^{*}$ in $D \times \mathbf{P}^{1}(\mathbf{C})$ and $\tilde{M}$ the universal covering of $\hat{M}$. Then $\tilde{M}$ is canonically regarded as a covering surface over $D$ with the projection $F: \tilde{M} \rightarrow D$.

Now, consider the many-valued meromorphic function

$$
\tilde{g}(\zeta):=(\phi \cdot F)^{1 / 3}(\zeta)
$$

on $\tilde{M}$. By the definition of $\tilde{M}$, $\tilde{g}$ has single-valued branch on $\tilde{M}$, by which we define the function $g$. We next consider the many-valued meromorphic function

$$
\tilde{f}(\zeta):=\frac{F^{\prime}(\zeta)}{\left((\phi \cdot F)(\zeta)-a_{1}\right)^{1 / 3}\left((\phi \cdot F)(\zeta)-a_{2}\right)^{1 / 3}} .
$$

Since $(\phi \cdot F)-a_{1}$ and $(\phi \cdot F)-a_{2}$ have poles of order three at every point of $(\phi \cdot F)^{-1}(\infty), \tilde{f}$ has a single-valued branch on $\tilde{M}$, by which we define the function $f$. For these functions $f$ and $g$, we shall prove that the minimal surface $x=\left(x_{1}, x_{2}, x_{3}\right): \tilde{M} \rightarrow \mathbf{R}^{3}$ defined by (6.11) has all desired properties.

Take a reduced representation $g=g_{1} / g_{0}$, or $g=\left(g_{0}: g_{1}\right)$ as a map into $\mathbf{P}^{1}(\mathbf{C})$. The induced metric is given by

$$
d s^{2}=\frac{1}{4}|f|^{2}\left(1+|g|^{2}\right)^{2}|d \zeta|^{2}=\frac{|f|^{2}}{4\left|g_{0}\right|^{4}}\left(\left|g_{0}\right|^{2}+\left|g_{1}\right|^{2}\right)^{2}|d \zeta|^{2}
$$

Obviously, $\nu_{d s}=0$ at each point where $g$ has no zero or no pole. The same is true at each point where $g$ has a pole, because $g_{0}$ has a zero of order one and the denominator of the right hand side of (6.12) has a pole of order two at such a point. Moreover, $\nu_{d_{s}}(\zeta)=\nu_{F^{\prime}}(\zeta)=2$ whenever $g(\zeta)=0$. These show that $\nu_{d s}=2 \nu_{g_{1}}$. If we consider a bounded function $k:=\left|g_{1}\right|^{2} /\left(\left|g_{0}\right|^{2}+\left|g_{1}\right|^{2}\right)(\leq 1)$, we obtain

$$
\left[\nu_{d s}\right]=d d^{c} \log \left|g_{1}\right|^{4}=2 d d^{c} \log \|g\|^{2}+d d^{c} \log k^{2} .
$$


This gives $\rho_{d s} \leq 2$.

We next show that $\tilde{M}$ is complete. Assume that there is a curve $\Gamma$ in $\tilde{M}$ which is of finite length and tends to the boundary. For $\Gamma^{\prime}:=F(\Gamma)$ in $D$ we have

$$
\begin{aligned}
L:=L_{d s}(\Gamma) & =\int_{\Gamma \mid} \frac{\left|F^{\prime}(\zeta)\right|\left(1+|(\phi \cdot F)(\zeta)|^{2 / 3}\right)}{\left|(\phi \cdot F)(\zeta)-a_{1}\right|^{1 / 3}\left|(\phi \cdot F)(\zeta)-a_{2}\right|^{1 / 3}}|d \zeta| \\
& =\int_{\Gamma^{\prime}} \frac{1+|\phi(z)|^{2 / 3}}{\left|\phi(z)-a_{1}\right|^{1 / 3}\left|\phi(z)-a_{2}\right|^{1 / 3}}|d z|
\end{aligned}
$$

and $\Gamma^{\prime}$ tends to the set $\partial D=\{\infty\} \cup \phi^{-1}\left(a_{1}\right) \cup \phi^{-1}\left(a_{2}\right)$. On the other hand, we can easily find a positive constant $C_{0}$ such that

$$
\frac{1+|\phi(z)|^{2 / 3}}{\left|\phi(z)-a_{1}\right|^{1 / 3}\left|\phi(z)-a_{2}\right|^{1 / 3}} \geq C_{0}
$$

for every $z \in D$. If $\Gamma^{\prime}$ tends to $\infty$, then we get an absurd conclusion

$$
L \geq C_{0} \int_{\Gamma^{\prime}}|d z|=+\infty \text {. }
$$

Otherwise, there exists some point $z_{0} \in \phi^{-1}\left(a_{1}\right) \cup \phi^{-1}\left(a_{2}\right)$ such that $\Gamma^{\prime}$ tends to $z_{0}$. Changing indices if necessary, we may assume that $\phi\left(z_{0}\right)=a_{1}$. Then, we find a neighborhood $U$ of $z_{0}$ and a positive constant $C_{1}$ such that the following inequality holds on $U$

$$
\frac{1+|\phi(z)|^{2 / 3}}{\left|\phi(z)-a_{1}\right|^{1 / 3}\left|\phi(z)-a_{2}\right|^{1 / 3}} \geq C_{1} \frac{1}{\left|z-z_{0}\right|},
$$

because $\nu_{\phi-\alpha_{1}}\left(z_{0}\right)=3$. For the portion $\tilde{\Gamma}$ of $\Gamma^{\prime}$ in $U$, we obtain

$$
L \geq \int_{\tilde{\Gamma}} \frac{1+|\phi(z)|^{2 / 3}}{\left|\phi(z)-a_{1}\right|^{1 / 3}\left|\phi(z)-a_{2}\right|^{1 / 3}}|d z| \geq C_{1} \int_{\tilde{\Gamma}} \frac{|d z|}{\left|z-z_{0}\right|}=+\infty .
$$

This concludes the completeness of $\tilde{M}$.

On the other hand, since $\phi(z) \neq a_{1}, a_{2}$ on $D$, we see

$$
g(\zeta) \notin\left\{a_{1}^{1 / 3}, a_{1}^{1 / 3} \omega, a_{1}^{1 / 3} \omega^{2}, a_{2}^{1 / 3}, a_{2}^{1 / 3} \omega, a_{2}^{1 / 3} \omega^{2}\right\}
$$

for every $\zeta \in \tilde{M}$, where $\omega$ denotes one of the primitive third roots of unity. So, the modified defects of these values are all one. This shows that the inequality (6.9) is best-possible in this case. 


\section{REFERENCES}

[1] L. A. Ahlfors, Conformal invariants, Topics in Geometric Function Theory, McGraw Hill, New York, 1973.

[2] W. Chen, Cartan's conjecture: Defect relation for meromorphic maps from parabolic manifold to projective space, Notre Dame Dissertation, 1987.

[ 3 ] S. S. Chern and R. Osserman, Complete minimal surfaces in euclidean $n$-space, J. Analyse Math., 19 (1967), 15-34.

[4] M. J. Cowen and P. A. Griffiths, Holomorphic curves and metrics of negative curvature, J. Analyse Math., 29 (1976), 93-153.

[5] H. Fujimoto, On the number of exceptional values of the Gauss map of minimal surfaces, J. Math. Soc. Japan, 40 (1988), 235-247.

[6] _- Examples of complete minimal surfaces in $\mathbf{R}^{m}$ whose Gauss maps omit $m(m+1) / 2$ hyperplanes in general position, Sci. Rep. Kanazawa Univ., 33 (1988), $37-43$.

[ 7 ] - Modified defect relations for the Gauss map of minimal surfaces, J. Differential Geom., 29 (1989), 245-262.

[ 8 ] — Modified defect relations for the Gauss map of minimal surfaces. II, J. Differential Geom., 31 (1990), 365-385.

[ 9 ] W. K. Hayman, Meromorphic functions, Oxford Math. Monographs, Clarendon Press, Oxford, 1964.

[10] A. Huber, On subharmonic functions and differential geometry in the large, Comment. Math. Helv., 32 (1957), 13-72.

[11] X. Mo and R. Osserman, On the Gauss map and total curvature of complete minimal surfaces and an extension of Fujimoto's theorem, J. Differential Geom., 31 (1990), 343-355.

[12] E. I. Nochka, On the theory of meromorphic functions, Soviet Math. Dokl., 27 (2) (1983).

[13] M. Ru, On the Gauss map of minimal surfaces immersed in $\mathbf{R}^{m}$, preprint.

[14] B. V. Shabat, Distribution of values of holomorphic mappings, Transl. Math. Monographs Vol. 61, AMS, 1985.

[15] M. Tsuji, Potential theory in modern function theory, Maruzen Tokyo, 1959.

[16] B. White, Complete surfaces of finite total curvature, J. Differential Geom., 26 (1987), 315-326.

Department of Mathematics

Faculty of Science

Kanazawa University

Kanazawa, 920

Japan 